\title{
nuSTORM: Neutrinos from Stored Muons
}

\author{
F.J.P. Soler ${ }^{1}$, \\ School of Physics and Astronomy, \\ University of Glasgow, \\ Glasgow, G12 8QQ, UK
}

To appear in the proceedings of the Prospects in Neutrino Physics Conference, 15 - 17 December, 2014, held at Queen Mary University of London, UK.

\section{Introduction}

Decay rings for muons were proposed by Koshkarev in 1974 [1] and Neuffer in 1980 [2]. This led to developments of the modern concept of a Neutrino Factory, which may be seen as a first step towards a Muon Collider. The physics capabilities of a Neutrino Factory were described by Geer [3]. The neutrino beam is created from the decay of muons in flight in a storage ring, and may be used to discover $\mathrm{CP}$ violation in the neutrino sector and to resolve the neutrino mass ordering. Since both $\mu^{+}$and $\mu^{-}$can be created with the same systematic uncertainties on the flux, any oscillation channel can be studied with both neutrinos and antineutrinos, improving sensitivity to $\mathrm{CP}$ violation.

A simple first step towards a Neutrino Factory could be the nuSTORM (Neutrinos from STORed Muons) decay ring. The main motivation for nuSTORM is to design and construct a new type of neutrino beam with well-understood characteristics from decays of muons that does not require any new technology, such as ionisation cooling. The facility consists of a $3.8 \mathrm{GeV} / \mathrm{c}$ muon storage ring that can be used to study eV-scale neutrino oscillation physics, $\nu_{e}$ and $\nu_{\mu}$ interaction physics and to develop technology for future accelerator projects. The facility can search for sterile neutrinos in both appearance and disappearance modes, provide precise studies of electron and muon neutrino scattering on nuclei, in an energy appropriate for future long- and short-baseline neutrino oscillation programmes, and provide the technology test-bed required to carry-out the $\mathrm{R} \& \mathrm{D}$ critical for the implementation of the next step in a muon-accelerator based particle-physics programme.

\section{The nuSTORM concept}

The nuSTORM facility consists of a ring designed to store muons with momenta of $3.8 \mathrm{GeV} / \mathrm{c}$. Protons of $120 \mathrm{GeV} / \mathrm{c}$ are used to produce pions from a conventional solid target. The pions are collected with a magnetic horn and quadrupole magnets and those with $5 \mathrm{GeV} / \mathrm{c}$ momentum are injected into a storage ring. The pions that decay in the first straight of the

\footnotetext{
${ }^{1}$ On behalf of the nuSTORM Collaboration
} 
ring yield muons, and those with momenta of $3.8 \mathrm{GeV} \pm 10 \%$ are captured in the storage ring. The circulating muons then subsequently decay into electrons and neutrinos. The momentum was selected to maximise the physics reach for both neutrino oscillations and to measure neutrino cross sections in an energy relevant for long-baseline neutrino oscillation experiments. See Figure 1 for a schematic of the facility. More details of the nuSTORM facility may be found in References [4, 5, 6].

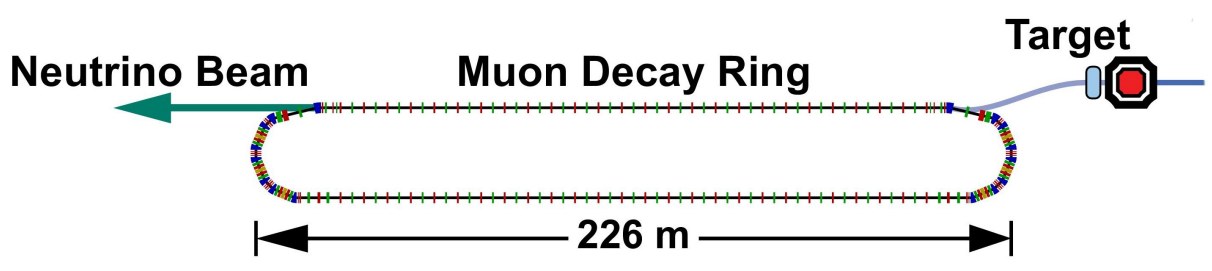

Figure 1: A schematic of the storage ring configuration. Pions are injected into a straight section and must decay into muons before the first bend or be ejected from the ring. Muons that decay in the injection straight during subsequent turns produce the neutrino beam.

\subsection{Physics motivation of nuSTORM}

A number of results have been reported that can be interpreted as hints for oscillations involving sterile neutrinos with masses at the eV scale (for a recent review see [7]). nuSTORM is capable of making the measurements required to confirm or refute the evidence for sterile neutrinos using a technique that is both qualitatively and quantitatively new. The nuSTORM facility delivers beams of $\nu_{e}\left(\bar{\nu}_{e}\right)$ and $\bar{\nu}_{\mu}\left(\nu_{\mu}\right)$. A detector located at a distance $\sim 2000 \mathrm{~m}$ from the end of one of the straight sections will be able to make sensitive searches for the existence of sterile neutrinos. If no appearance $\left(\nu_{e} \rightarrow \nu_{\mu}\right)$ signal is observed, the allowed region can be ruled out at the $\sim 10 \sigma$ level [8]. Instrumenting the nuSTORM neutrino beam with a near detector at a distance of $\sim 20$ m makes it possible to search for sterile neutrinos in the disappearance $\nu_{e} \rightarrow \nu_{X}$ and $\bar{\nu}_{\mu} \rightarrow \bar{\nu}_{X}$ channels. In the disappearance search, the absence of a signal would permit the presently allowed region to be excluded at the $99 \%$ confidence level. For a general discussion of the optimisation of disappearance searches at short baselines see [9].

With the discovery that $\theta_{13}$ is non-zero [10, 11, 12, 13, 14] and that it has a larger than expected value $\left(\sin ^{2} 2 \theta_{13} \sim 0.1\right)$, the search for $\mathrm{CP}$ violation in the lepton sector requires measurements of oscillation probabilities with uncertainties at the percent level. For future long-baseline experiments to reach their ultimate precision requires that the $\nu_{e} N$ and the $\nu_{\mu} N$ cross sections are known precisely for neutrino energies $\left(E_{\nu}\right)$ in the range $0.5<E_{\nu}<3 \mathrm{GeV}$. nuSTORM is unique as it makes it possible to measure the $\bar{\nu}_{\mu}\left(\nu_{\mu}\right)$ and $\nu_{e}\left(\bar{\nu}_{\mu}\right)$ nucleus cross sections with a precision $\simeq 1 \%$ over the required neutrino-energy range. The flavour composition of the beam and the neutrino-energy spectrum are both precisely known. The storage-ring instrumentation combined with measurements at a near 
detector will allow the neutrino flux to be determined with a flux accuracy of $\simeq 10^{-3}$, with the potential to transform the field of neutrino interaction physics.

Finally, the unique capabilities of nuSTORM offer the opportunity to provide muon beams for future investigations into six dimensional muon ionisation cooling, while running the neutrino programme simultaneously. Muon cooling is the key enabling technology needed for future ultra-high intensity muon accelerator facilities. Its demonstration would be one of the major steps towards the realisation of a multi-TeV Muon Collider.

\section{$2.2 \quad$ Long baseline physics}

Neutrino Factories have demonstrated the best potential sensitivity for CP violation of all future long-baseline neutrino oscillation facilities. To measure the $\mathrm{CP}$ asymmetry $\left(A_{C P}\right)$ at $3 \sigma$ for $75 \%$ coverage of the values of $\delta_{C P}$, means that $A_{C P}$ may be as low as $5 \%$, which requires a precision of $1.5 \%$ and a systematic error of order $1 \%$. However, we measure the rate:

$$
R_{\alpha \beta}\left(E_{v i s}\right)=N \int d E \Phi_{\alpha}(E) \sigma_{\beta}\left(E, E_{v i s}\right) \epsilon_{\beta}(E) P\left(\nu_{\alpha} \rightarrow \nu_{\beta}, E\right),
$$

where $E$ is the neutrino energy, $E_{v i s}$ is the visible energy, $\Phi_{\alpha}(E)$ is the flux of $\nu_{\alpha}, \sigma_{\beta}\left(E, E_{v i s}\right)$ is the cross section and $\epsilon_{\beta}$ is the efficiency of detection of a $\nu_{\beta}$ of energy $E$ but measured at a visible energy $E_{v i s}$, and $P\left(\nu_{\alpha} \rightarrow \nu_{\beta}, E\right)$ is the probability of oscillation from $\nu_{\alpha}$ to $\nu_{\beta}$ [15]. In a disappearance experiment, we can satisfy:

$$
\frac{R_{\alpha \beta}(\text { far }) L^{2}}{R_{\alpha \beta}(\text { near })} \sim \frac{N_{\text {far }} \Phi_{\alpha} \sigma_{\beta} \epsilon_{\beta} P\left(\nu_{\alpha} \rightarrow \nu_{\beta}\right)}{N_{\text {near }} \Phi_{\alpha} \sigma_{\alpha} \epsilon_{\alpha}},
$$

where $\alpha=\beta$, so many of the systematic errors cancel. However, in an appearance experiment, $\alpha \neq \beta$, so the $\nu_{\alpha}$ beam cannot be used to measure $\sigma_{\beta} \epsilon_{\beta}$. The difference in the

$\sigma_{\nu_{e}}$ and $\sigma_{\nu_{\mu}}$ cross sections can be large, depending on the energy, due to nuclear effects and this may affect the neutrino oscillation measurements. For example, in the Tokai to Hyper-Kamiokande (T2HK) proposed experiment, with a precision in the ratio of cross sections $\sigma_{\nu_{e}} / \sigma_{\nu_{\mu}}$ between $1 \%$ and $2 \%$ one can achieve a $3 \sigma$ measurement of $\mathrm{CP}$ violation for $75 \%$ of the $\delta_{C P}$ values with an exposure between 600 and $800 \mathrm{kton} \cdot \mathrm{MW} \cdot$ years. However, a degradation of the systematic uncertainty to the $5 \%$ level corresponds to an increase in the exposure of roughly 200-300\% required to achieve $3 \sigma$ accuracy for $75 \%$ of the $\delta_{C P}$ values [16]. Muon storage rings, such as nuSTORM or a Neutrino Factory, are the only known facilities that can achieve a measurement of cross sections with less than $1 \%$ precision.

\subsection{Short baseline physics}

The LSND [17, 18] and MiniBooNE experiments [19, 20] show hints of $\bar{\nu}_{\mu} \rightarrow \bar{\nu}_{e}$ and $\nu_{\mu} \rightarrow \nu_{e}$ appearance, that can be explained by an oscillation mediated by sterile neutrino states, in short-baseline accelerator experiments. Furthermore, there is additional evidence of a $6 \%$ deficit of $\bar{\nu}_{e}$ from reactor experiments (the reactor anomaly) based on more accurate recent re-evaluations of the reactor antineutrino flux [21, 22, 23, 12] that can also be interpreted 
as antineutrino disappearance via sterile neutrino mediated oscillations. Finally, active-tosterile neutrino oscillations can also explain the gallium anomaly, in which intense artificial radioactive sources used to calibrate gallium radiochemical detection experiments observed fewer neutrinos from the source than expected [24, 25].

Global fits attempt to explain these data, but there exists tension between the appearance and disappearance measurements [26]. The sterile neutrino hypothesis is satisfied when:

$$
P\left(\nu_{\mu} \rightarrow \nu_{e}\right) \leq 4\left(1-P\left(\nu_{\mu} \rightarrow \nu_{\mu}\right)\right)\left(1-P\left(\nu_{e} \rightarrow \nu_{e}\right)\right) .
$$

The nuSTORM facility could probe all possible sterile neutrino appearance and disappearance channels to test the sterile neutrino paradigm in detail.

\section{3 nuSTORM parameters}

The nuSTORM facility is designed to produce $3.8 \mathrm{GeV} / \mathrm{c}$ muons that are injected and stored in a storage ring (Figure 1). A $100 \mathrm{~kW}$ proton beam of $120 \mathrm{GeV}$ energy impinges on a carbon or an inconel target. Pions produced in the target are captured in a NuMI-style horn, they are then transported down a transfer line and $5 \mathrm{GeV} / \mathrm{c}( \pm 20 \%)$ pions are stochastically injected into a storage ring. The target, collection system and stochastic injection systems have been designed to deliver 0.11 pions per proton on target (POT) [4] to the storage ring.

The storage ring consists of a large aperture FODO lattice designed to transport muons of $3.8 \mathrm{GeV} / \mathrm{c}( \pm 10 \%)$ momenta around the ring. It is calculated that $52 \%$ of pions decay to muons before the first turn and $8 \times 10^{-3}$ muons per POT are stored in the storage ring. For $10^{20}$ POT, we expect a flash of neutrinos from $8.6 \times 10^{18}$ pion decays and we expect $2.6 \times 10^{17}$ positive muons that decay in the ring (the muon lifetime is 27 orbits of the decay ring). The nuSTORM flux and energy spectrum from the pion flash and from recirculating muons are shown in Figure 2,
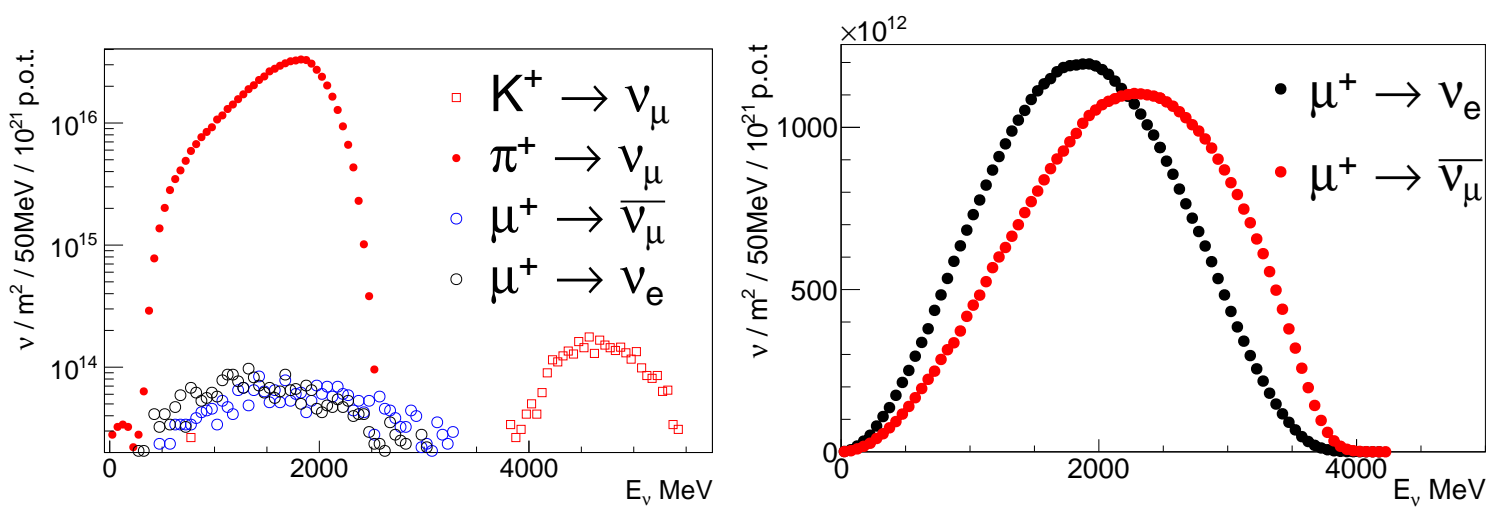

Figure 2: nuSTORM flux and energy spectrum from the pion flash just after injection (left) and from muon decay over 100 turns (right).

The flux of $\nu_{\mu}$ from pion decays is $6.3 \times 10^{16} \nu / \mathrm{m}^{2}$, the flux of $\nu_{e}$ from muon decays is $3.0 \times 10^{14} \nu / \mathrm{m}^{2}$ and $\nu_{\mu}$ from kaon decay is $3.8 \times 10^{14} \nu / \mathrm{m}^{2}$, all at a distance of $50 \mathrm{~m}$. 
This hybrid beam from pion and muon decay can produce a rich physics programme of neutrino cross-section measurements and can be used to perform a sterile neutrino search at a short-baseline oscillation experiment. Furthermore, the flux uncertainties for nuSTORM are less than $1 \%$, due to the precise knowledge of the muon decay spectrum and from the instrumentation that can be installed in the storage ring to measure the number of muons in the ring. The event rates per $10^{21}$ POT in 100 tons of a Liquid Argon detector at $50 \mathrm{~m}$ are shown in Table 1 .

Table 1: Event rates per $10^{21}$ POT in 100 tons of Liquid Argon at $50 \mathrm{~m}$ from the nuSTORM storage ring.

\begin{tabular}{|cc|cc|}
\hline$\mu^{+}$Channel & $N_{\text {evts }}$ & $\mu^{-}$Channel & $N_{\text {evts }}$ \\
\hline $\bar{\nu}_{\mu} \mathrm{NC}$ & $1,174,710$ & $\bar{\nu}_{e} \mathrm{NC}$ & $1,002,240$ \\
$\nu_{e} \mathrm{NC}$ & $1,817,810$ & $\nu_{\mu} \mathrm{NC}$ & $2,074,930$ \\
$\bar{\nu}_{\mu} \mathrm{CC}$ & $3,030,510$ & $\bar{\nu}_{e} \mathrm{CC}$ & $2,519,840$ \\
$\nu_{e} \mathrm{CC}$ & $5,188,050$ & $\bar{\nu}_{\mu} \mathrm{CC}$ & $6,060,580$ \\
\hline$\pi^{+}$Channel & $N_{\text {evts }}$ & $\pi^{-}$Channel & $N_{\text {evts }}$ \\
\hline$\nu_{\mu} \mathrm{NC}$ & $14,384,192$ & $\bar{\nu}_{\mu} \mathrm{NC}$ & $6,986,343$ \\
$\nu_{\mu} \mathrm{CC}$ & $41,053,300$ & $\bar{\nu}_{\mu} \mathrm{CC}$ & $19,939,704$ \\
\hline
\end{tabular}

\section{Neutrino interaction physics}

There is a very rich physics programme in neutrino interaction physics that may be performed at the nuSTORM facility, due to its very large event rate and its accurate flux and energy determination. It will be able to perform crucial $\nu_{\mu}, \nu_{e}, \bar{\nu}_{\mu}$ and $\bar{\nu}_{e}$ cross-section measurements, required for the long-baseline neutrino oscillation programmes, with statistical and systematic uncertainties of less than $1 \%$, using the storage-ring instrumentation.

A number of near detectors are being considered for the facility, such as a detector similar to the HiResM $\nu$ detector proposed for DUNE at LBNF [27]. A high pressure gaseous or a liquid argon time projection chamber (TPC) would also be suitable choices. The performance of the HiResM $\nu$ detector exposed to the nuSTORM flux was studied in [5]. The charged-current quasi-elastic (CCQE) cross sections are plotted as a function of neutrino energy in Figure 3. The figure shows the precision with which the cross sections would be measured if the systematic uncertainties estimated for the HiResM $\nu$ detector are combined with the $1 \%$ flux uncertainty that nuSTORM will provide, compared to a flux uncertainty of $10 \%$. Figure 3 also shows the present measurements of the CCQE cross sections (only available for muon-neutrino and muon-anti-neutrino beams). The nuSTORM facility has the potential to improve the systematic uncertainty on $\nu_{\mu}$ and $\bar{\nu}_{\mu}$ CCQE cross section measurements by a factor of $5-6$. It is only very recently that preliminary results on $\nu_{e}$ CCQE cross section measurements are available [28] so nuSTORM will be able to perform comprehensive measurements with both $\nu_{e}$ and $\bar{\nu}_{e}$ beams.

Further to the potential to perform world-leading CCQE measurements, nuSTORM 
will probe other neutrino scattering topics, such as $\pi^{0}$ production in neutrino interactions, charged pion and kaon production, inclusive charged current and neutral currents, including the ratio of these, which can be used to determine $\sin ^{2} \theta_{W}$. It will be able to probe nuclear effects in neutrino interactions and other semi-exclusive and exclusive processes such as measurements of $K_{s}, \Lambda$ and $\bar{\Lambda}$ production. The nuSTORM facility can be used to search for new physics effects and exclusive processes, such as tests of $\nu_{\mu}$ and $\nu_{e}$ universality, searches for heavy neutrinos and eV-scale pseudo-scalar penetrating particles. In summary, there exists a very rich physics programme to be carried out at a near detector at the nuSTORM facility that has the potential to revolutionise neutrino interaction physics.
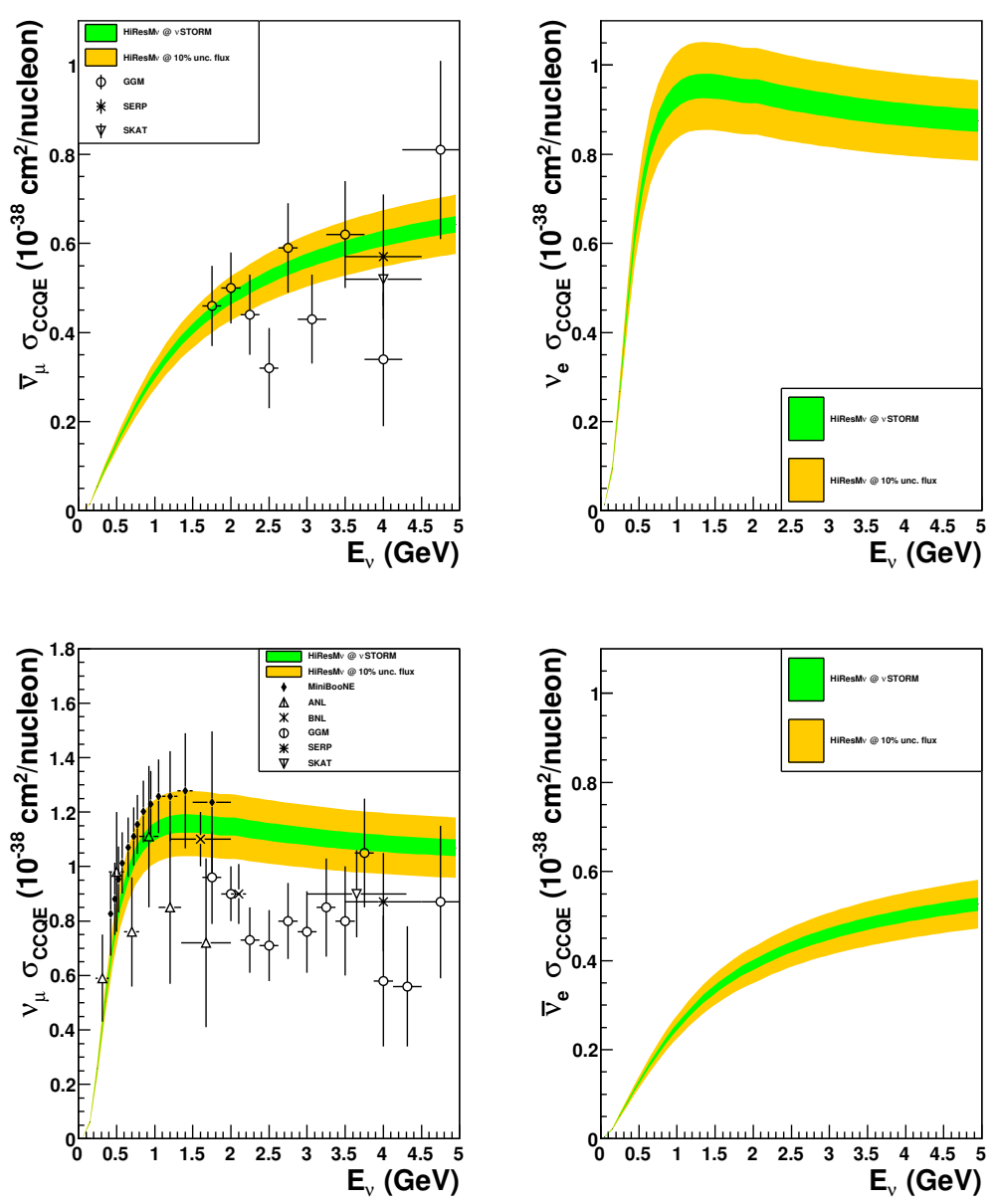

Figure 3: The CCQE cross sections $\left(\sigma_{\mathrm{CCQE}}\right)$ plotted as a function of incident neutrino energy $\left(E_{\nu}\right)$ for $\bar{\nu}_{\mu}$ (top, left), $\nu_{e}$ (top, right), $\nu_{\mu}$ (bottom, left) and $\bar{\nu}_{e}$ (bottom, right) interactions with the HiResM $\nu$ detector at nuSTORM (the green band includes $1 \%$ flux uncertainty and the yellow band shows $10 \%$ flux uncertainty). 


\section{$5 \quad$ Sterile neutrino search}

With the event rates expected at nuSTORM, one can carry out a sterile neutrino search in a short-baseline experiment, with a far detector, consisting of a 1.3 kton magnetised iron detector (Super BIND) at $2 \mathrm{~km}$, and a toroidal magnetic field between 1.5-2.6 T, fed by a superconducting transmission line delivering $240 \mathrm{kA}$-turns. A neutrino oscillation experiment can be carried out at the nuSTORM facility in which one can carry out simultaneously a $\nu_{\mu}$ appearance search and a $\bar{\nu}_{\mu}$ disappearance search. The probability of observing a $\nu_{e} \rightarrow \nu_{\mu}$ transition is given by

$$
P_{e \mu}=\sin ^{2} 2 \theta_{e \mu} \sin ^{2}\left(\frac{\Delta m^{2} L}{4 E}\right)
$$

where $\theta_{e \mu}$ is the effective mixing angle, and $\Delta m^{2}$ is the effective mass difference, independent of the sterile neutrino model. In the $(3+1)$ model (with only one sterile neutrino) then

$$
\sin ^{2} 2 \theta_{e \mu} \equiv 4\left|U_{\mu 4}\right|^{2}\left|U_{e 4}\right|^{2}
$$

where $U_{\epsilon n}$ is an element of the PMNS mixing matrix. The $\nu_{\mu}$ disappearance probability is

$$
P_{\mu \mu}=4\left|U_{\mu 4}\right|^{2}\left(1-\left|U_{\mu 4}\right|^{2}\right) \sin ^{2}\left(\frac{\Delta m^{2} L}{4 E}\right) .
$$

A complete appearance and disappearance analysis was carried out to determine the sensitivity of nuSTORM to search for eV-scale sterile neutrinos [8]. The short-baseline oscillation search was carried out by simulating a near detector at $50 \mathrm{~m}$ and a far detector at $2 \mathrm{~km}$, with a $10^{21}$ proton-on-target exposure. The appearance and disappearance analyses were carried out with two optimised multi-variate analyses. The search signal and background efficiencies are shown in Figure 4 and the sterile neutrino sensitivities also for the appearance and disappearance searches are shown in Figure 5, nuSTORM can either discover or rule out previous sterile neutrino evidence with better than $10 \sigma$ sensitivity.
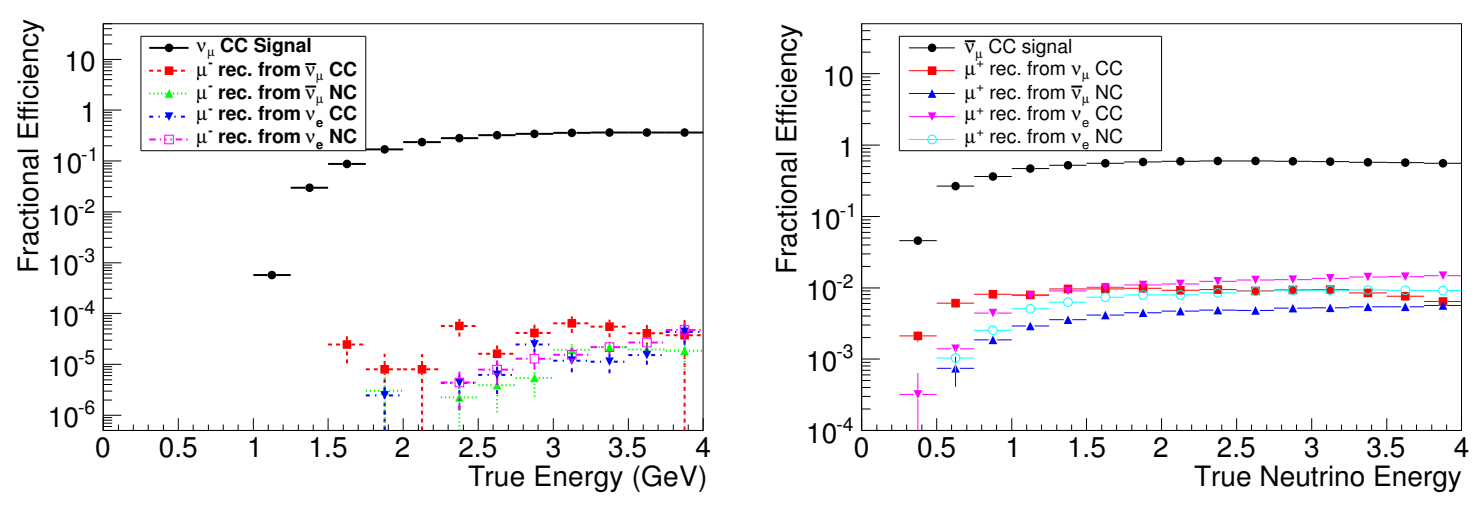

Figure 4: Efficiencies of signals and backgrounds for a $\nu_{\mu}$ appearance (left) and a $\bar{\nu}_{\mu}$ disappearance (right) neutrino oscillation search at nuSTORM. 

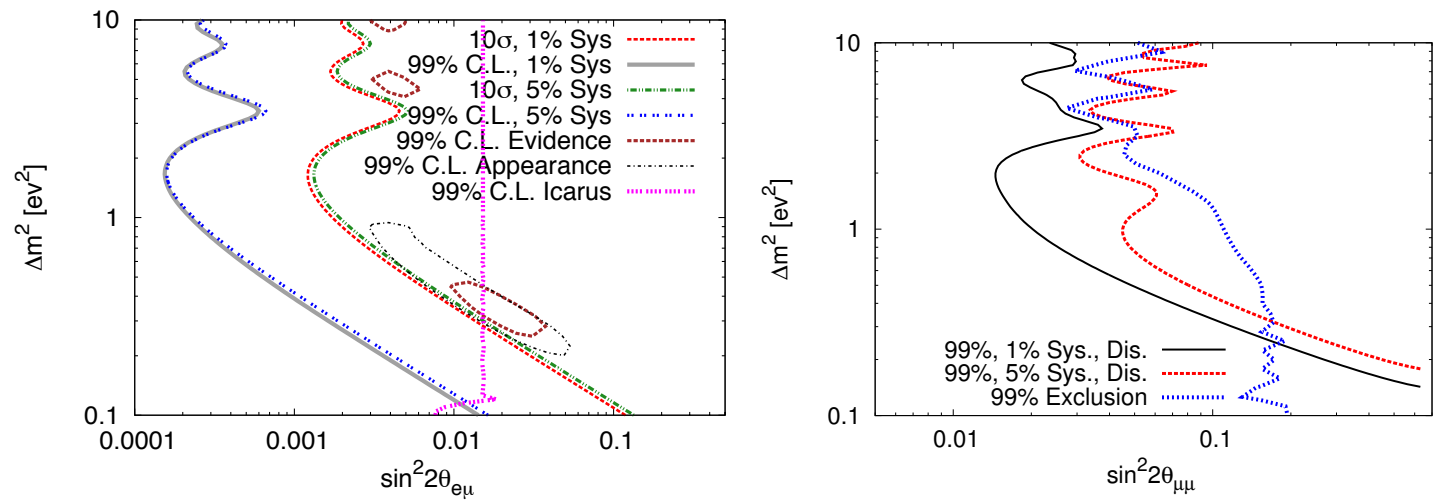

Figure 5: Sterile neutrino appearance channel sensitivity (left) at nuSTORM compared to 99\% confidence contours from fits generated by Kopp et. al. [26] and limits set by ICARUS [29]. Sensitivity of nuSTORM to $\bar{\nu}_{\mu}$ disappearance (right) oscillations assuming a $(3+1)$ neutrino model, compared to the existing disappearance data [26].

\section{Conclusions and Outlook}

The nuSTORM facility is an entry level Neutrino Factory that can be built now, without need of new technology such as ionisation cooling. It can carry out world-leading neutrino scattering physics studies, with the potential to measure all neutrino cross sections with high precision due to the less than $1 \%$ uncertainty on the knowledge of the flux. nuSTORM has the potential to resolve the issue of systematic errors for long-baseline neutrino oscillation experiments searching for CP violation. Hence, the nuSTORM facility would be the ultimate neutrino scattering physics facility: "a neutrino light source".

Furthermore, the nuSTORM facility could serve as a test-bed for muon acceleration $\mathrm{R} \& \mathrm{D}$, to develop the capabilities of muon storage rings for future particle physics projects. At the end of the first straight, one can place a $3.5 \mathrm{~m}$ iron pion absorber. After the absorber, $\sim 10^{10}$ muons per pulse can be observed between $100-300 \mathrm{MeV} / \mathrm{c}$. This high intensity muon source can be used to carry out a six-dimensional muon cooling experiment, essential to be able to realise future Neutrino Factories and Muon Colliders.

The nuSTORM facility could be sited at either Fermilab or in the North Area at CERN. A world-wide neutrino programme in which long-baseline experiments are carried out at LBNF in the USA and at Hyper-Kamiokande in Japan, could be complemented by the nuSTORM facility, which could be sited either at Fermilab or CERN to ensure that maximum impact is delivered from this programme. Therefore, the question should not be whether we can afford nuSTORM, but rather, whether we can afford not to have nuSTORM as a facility to perform high precision neutrino studies. 


\section{References}

[1] Koshkarev D G 1974 Proposal for a Decay Ring to Produce Intense Secondary Particle Beams at the SPS CERN/ISR-DI/74-62

[2] Neuffer D 1980 Design considerations for a muon storage ring telmark Conference on Neutrino Mass, Barger and Cline eds., Telmark, Wisconsin, TELE-1980-040, C801002

[3] Geer S 1998 Phys. Rev. D57 6989-6997 (Preprint hep-ph/9712290)

[4] Kyberd P et al. (nuSTORM Collaboration) 2012 (Preprint 1206.0294)

[5] Adey D et al. (nuSTORM Collaboration) 2013 (Preprint 1308.6822)

[6] Adey D, Agarwalla S, Ankenbrandt C, Asfandiyarov R, Back J et al. 2013 (Preprint 1305.1419)

[7] Abazajian K, Acero M, Agarwalla S, Aguilar-Arevalo A, Albright C et al. 2012 (Preprint 1204.5379)

[8] Adey D et al. (nuSTORM Collaboration) 2014 Phys.Rev.D (Preprint 1402.5250)

[9] Winter W 2012 Phys.Rev. D85 113005 (Preprint 1204.2671)

[10] An F et al. (DAYA-BAY Collaboration) 2012 Phys.Rev.Lett. 108171803 (Preprint 1203.1669)

[11] Ahn J et al. (RENO collaboration) 2012 Phys.Rev.Lett. 108191802 (Preprint 1204. 0626)

[12] Abe Y et al. (DOUBLE-CHOOZ Collaboration) 2012 Phys.Rev.Lett. 108131801 (Preprint 1112.6353)

[13] Abe K et al. (T2K Collaboration) 2011 Phys.Rev.Lett. 107041801 (Preprint 1106. 2822)

[14] Adamson P et al. (MINOS Collaboration) 2011 Phys.Rev.Lett. 107181802 (Preprint 1108.0015)

[15] Huber P, Bross A and Palmer M 2014 (Preprint 1411.0629)

[16] Huber P, Mezzetto M and Schwetz T 2008 JHEP 03021 (Preprint 0711.2950)

[17] Athanassopoulos C et al. (LSND) 1996 Phys. Rev. Lett. 77 3082-3085 (Preprint nucl-ex/9605003)

[18] Aguilar A et al. (LSND) 2001 Phys. Rev. D 64112007 (Preprint 0104049)

[19] Aguilar-Arevalo A A et al. (MiniBooNE) 2010 Phys. Rev. Lett. 105181801 (Preprint 1007.1150) 
[20] Aguilar-Arevalo A A et al. (MiniBooNE Collaboration) 2013 Phys.Rev.Lett. 110161801 (Preprint 1207.4809)

[21] Mueller T A et al. 2011 Phys. Rev. C83 054615 (Preprint 1101.2663)

[22] Mention G, Fechner M, Lasserre T, Mueller T A, Lhuillier D, Cribier M and Letourneau A 2011 Phys. Rev. D83 073006 (Preprint 1101.2755)

[23] Huber P 2011 Phys. Rev. C84 024617 [Erratum: Phys. Rev.C85,029901(2012)] (Preprint 1106.0687)

[24] Acero M A, Giunti C and Laveder M 2008 Phys. Rev. D78 073009 (Preprint 0711. 4222)

[25] Giunti C, Laveder M, Li Y F, Liu Q Y and Long H W 2012 Phys. Rev. D86 113014 (Preprint 1210.5715)

[26] Kopp J, Machado P A N, Maltoni M and Schwetz T 2013 JHEP 1305050 (Preprint 1303.3011)

[27] Mishra S R, Petti R and Rosenfeld C 2008 PoS NUFACT08 069 (Preprint 0812.4527)

[28] Wolcott J (MINERvA) 2015 PoS NUFACT2014 058 (Preprint 1501.05214)

[29] Antonello M et al. 2013 Eur.Phys.J. C73 2345 (Preprint 1307.4699) 